\title{
Seguimento de pessoas acometidas por doenças cerebrovasculares em um hospital regional amazônico: uma metodologia do WHO STEPS Stroke
}

\section{Follow-up of people affected by cerebrovascular disease in a regional amazonic hospital: a WHO STEPS Stroke methodology}

(DTiótrefis Gomes Fernandes ${ }^{1}$, (DPedro Porto Alegre Baptista ${ }^{1}$, (D)onathas Gonçalves da Costa ${ }^{1}$, (DPaulo Andrade Lotufo ${ }^{2}$, (DTania Fernandes Campos ${ }^{3}$

${ }^{1}$ Universidade Federal do Amazonas - UFAM
${ }^{2}$ Universidade de São Paulo - USP

${ }^{3}$ Universidade Federal do Rio Grande do Norte UFRN

\section{Correspondência}

Tiótrefis Gomes Fernandes

E-mail: tiotrefis@ufam.edu.br

Submetido: 09 Novembro 2020 Aceito: 27 Janeiro 2021

Como citar

Fernandes TG, Baptista PPA, Costa JG, Lotufo PA, Campos TF. Seguimento de pessoas acometidas por doenças cerebrovasculares em um hospital regional amazônico: uma metodologia do WHO STEPS Stroke. Acta Fisiatr. 2020;27(4):206-212.

DOI: 10.11606/issn.2317-0190.v27i4a174718

\section{RESUMO}

O WHO STEP Stroke é uma ferramenta desenvolvida para monitorar casos de doenças cerebrovasculares e possibilitar comparações de incidências pelo mundo. Objetivo: Descrever o perfil sociodemográfico e a funcionalidade de pacientes acometidos por AVC admitidos no Hospital Regional de Coari durante um ano, e acompanha-los ao longo de um mês após admissão. Método: Estudo descritivo observacional, prospectivo e de seguimento dos casos entre outubro de 2010 e outubro de 2011 . Utilizou-se a primeira etapa (Step 1) do WHO STEP Stroke para avaliar as hospitalizações por AVC em Coari/AM. Foram acompanhados 23 pacientes através de questionário padrão em até dois dias na internação, 10 e 28 dias após a internação. Resultados: A população estudada tinha média de idade de $72,8(D P=12,4)$ anos, em sua maioria homens $(65,7 \%)$, pardos $(65,2 \%)$ e hipertensos $(95,7 \%)$, com incidência hospitalar de 30 casos em 100.000 habitantes e taxa de letalidade em 10 dias de 30,4\% e 28 dias de 34,8\%. O comprometimento neurológico na internação teve mediana de $28(I I Q=15-38)$ pontos, sendo $73,9 \%$ classificados como grave. Houve comprometimento funcional entre os sobreviventes. Conclusão: Este estudo revelou uma predominância de homens, com idade elevada, pardos, sem estudo formal, baixa renda e com histórico de hipertensão arterial e diabetes, como perfil de internação hospitalar por doenças cerebrovasculares no município. A incidência de casos hospitalares de AVC foi em 30 (por 100.000) em Coari-AM, com graves comprometimentos neurológicos na admissão, e altas taxas de letalidade e prejuízo funcional após 28 dias do evento, quando comparadas a outras localidades no Brasil e no mundo.

Palavras-chaves: Acidente Vascular Cerebral, Desempenho Físico Funcional, Epidemiologia, Brasil

\section{ABSTRACT}

The WHO STEP Stroke is a tool developed to monitor cases of cerebrovascular and allow comparison of its incidence throughout the world. Objective: To describe the sociodemographic profile and functionality of stroke inpatients admitted in Coari's Regional Hospital and followed up one month after admittance. Method: This is a prospective, descriptive observational and follow-up study of cases between October 2010 and October 2011. In this study, we used the first step (Step 1) of the WHO STEP Stroke to assess stroke hospitalization in Coari/AM Twenty-three patients were monitored with a standard questionnaire up to two days after hospitalization, 10 days, and 28 after hospitalization. Results: The studied population had average age of 72.8 (SD= 12.4) years, mostly men (65.7\%), brown (65.2\%), and hypertensive (95.7\%), with a hospital incidence of 30 cases for 100.000 people, and a mortality in 10 days of $30.4 \%$ and 28 days of $34.8 \%$. The neurological impairment at hospitalization had a median of 28 (IIQ= 15-38) points, with $73.9 \%$ classified as severe. There was an impairment in functionality among the survivors. Conclusion: This study showed the profile of hospital admittance for cerebrovascular disease in this city to be mainly composed by older men, brown, with no formal study, low income, and history of systemic arterial hypertension and diabetes. The hospital incidence for stroke was 30 (per 100.000) in Coari-AM, with severe neurological impairment at admission, with high mortality rates, and functional deficits 28 days after the event when compared to other regions in Brazil and in the world.

Keywords: Stroke, Physical Functional Performance, Epidemiology, Brazil 


\section{INTRODUÇÃO}

A doença cerebrovascular ou acidente vascular cerebral (AVC) é a principal causa de incapacidade no mundo e a terceira principal no Brasil. ${ }^{1,2}$ Relatórios da Organização Mundial da Saúde (WHO) têm apontado que o número de pessoas com incapacidades e diminuição de funcionalidade vêm aumentado significativamente nos últimos anos, devido ao aumento na expectativa de vida e da incidência de doenças não transmissíveis. ${ }^{3}$ A partir desta perspectiva, foi elaborado a metodologia WHO STEPS Stroke ("WHO STEPwise approach to Stroke Surveillance") com o objetivo de ampliar os conceitos relativos ao AVC e permitir a comparação de estudos de incidências da doença pelo mundo. ${ }^{4}$

O WHO STEPS Stroke é uma metodologia proposta em 2001 por Thomas Truelsen, Ruth Bonita e Konrad Jamrozik, ${ }^{5}$ utilizada pela WHO com o intuito de estimular a vigilância epidemiológica do AVC. A ferramenta é utilizada para avaliar o impacto da doença, descrever as populações de risco, monitorar e avaliar as intervenções. Dividida em três etapas, a primeira (Step 1) aborda os eventos de hospitalização de pacientes admitidos por AVC. O Step 1 coleta informações sociodemográficas e condições pré-clínicas destas pessoas, sendo possível obter o número de admissões, gravidade da doença, as taxas de letalidade e de incidência hospitalar.

A segunda etapa (Step 2) aborda os eventos fatais por AVC na comunidade. Nesta avalia-se as informações obtidas por meio das declarações de óbito, autópsias e/ou necropsias, a fim de determinar o tipo e subtipo de AVC. Na terceira etapa (Step 3) avalia-se os eventos não-fatais por AVC na comunidade. Aqui, a população de referência deve estar bem definida e então estima-se as taxas de letalidade e incidência do AVC e, associada as etapas 1 e 2, a prevalência. ${ }^{5}$ Esta abordagem dispensa equipamentos sofisticados e permite comparabilidade de resultados, sendo aplicável também em locais com poucos recursos e afastados de grandes centros urbanos, como o município de Coari, Amazonas.

Coari apresenta um território com quase $58 \mathrm{mil} \mathrm{km}^{2}$ e população com cerca de 76 mil habitantes em 2010 e estimativa de 85 mil habitantes em 2019, de acordo com o censo brasileiro de $2010,{ }^{6}$ e vem experimentando um aumento de sua urbanização nas últimas décadas. Em 2010, 65,4\% da população de Coari habitava em agrupamento urbano, enquanto $35,6 \%$ em comunidades ribeirinhas (área rural). A região conta somente com único hospital de média complexidade, o Hospital Regional de Coari (HRC), e 11 unidades de atenção primária - cobrindo $100 \%$ da população urbana, e duas unidades de saúde móvel (em barcos) para cobrir a área rural.

O HRC conta com 83 leitos, e configura-se como centro de referência do município e para outros cinco municípios próximos. Neste estabelecimento há quatro especialidades médicas (clínica médica, clínica cirúrgica, clínica ginecoobstétrica e clínica pediátrica), acrescida de ortopedia, atendendo também urgências e emergências.

Um estudo anterior neste município (Step 3 do estudo EMMA-Coari), realizado em 2011 com pessoas acima de 35 anos, encontrou uma prevalência de AVC de 3,7\% entre aqueles que vivem na área urbana e $6,3 \%$ entre os que vivem na área rural deste município. E dentre os casos, 33,3\% tiveram algum comprometimento funcional. ${ }^{7}$

A perda de funcionalidade é um fator de alto impacto na qualidade de vida de pacientes com AVC. ${ }^{8,9}$ Estima-se que cerca de $50 \%$ dos pacientes com AVC apresentem algum grau de incapacidade funcional após o evento, sendo a principal doença incapacitante nos Estados Unidos da América. ${ }^{10}$ Desta forma, a avaliação da incapacidade e da independência funcional é crucial para a compreensão do quadro clínico, sobretudo no primeiro mês após o evento. $E$ as ferramentas de medida de desfecho funcional mais utilizadas na população com AVC tem sido a Escala Modificada de Rankin (mRS) e a Medida de Independência Funcional (MIF). ${ }^{8,9,11,12}$

\section{OBJETIVOS}

O objetivo desta pesquisa é descrever o perfil sociodemográfico e a funcionalidade de pacientes acometidos por AVC admitidos no Hospital Regional de Coari durante um ano, e acompanhá-los ao longo de um mês após admissão.

\section{MÉTODOS}

O presente trabalho trata de um estudo descritivo observacional, prospectivo e de seguimento de casos hospitalares de AVC no município de Coari/AM, no período de outubro de 2010 a outubro de 2011. Este foi a realização do Step 1 do "Estudo de Morbidade e Mortalidade do Acidente Vascular Cerebral na cidade de Coari/AM" (EMMA-Coari) extensão do estudo EMMA-São Paulo. ${ }^{13} \mathrm{O}$ mesmo contou com a parceria do projeto "Programa de Diagnóstico e Intervenção no Acidente Vascular Cerebral (PRODIAVC)". ${ }^{14}$

A pesquisa foi desenvolvida no Hospital Regional de Coari (HRC), onde foram identificados todos os casos índices com diagnóstico clínico de doença cerebrovascular na admissão ou até dois dias de internação, sendo então submetidos ao questionário padrão recomendado pela WHO STEP Stroke. Não houve uma análise retrospectiva dos pacientes anteriormente internados, para evitar problemas com qualidade de registros hospitalares. O questionário padrão é composto por oito partes: Identificação do Paciente (condições sociodemográficas, como: idade, sexo, nível educacional, ocupação e renda familiar) e 7 módulos. Os módulos 1 a 3 versam sobre a avaliação do caso e, juntamente com os dados de identificação e a escala de avaliação clínica de AVC do Instituto Nacional do Estados Unidos - National Institute Health Stroke Scale (NIHSS), foram realizadas no primeiro momento após admissão hospitalar.

No módulo 1 estão as perguntas sobre o histórico clínico do paciente e sobre a presença de condições pré-clínicas: hipertensão, diabetes, cardiopatia, dependência de nicotina, consumo de álcool, SIDA, sífilis, tuberculose, câncer ou outro.

No módulo 2 estão as perguntas sobre o tratamento médico atual e sobre sua funcionalidade através da Escala de Atividade Básica de Vida Diária (ABVD) e da Escala de Rankin modificada (mRS); no módulo 3 estão as perguntas sobre o tipo de AVC.

Os módulos 4 e 5 foram realizados no 10 으 dia pós-AVC e contém perguntas sobre status vital e funcionalidade pela ABVD e mRS. Os módulos 6 e 7 foram realizados no 280 dia pósAVC e contém perguntas sobre status vital e funcionalidade pela ABDV e mRS, sendo acrescentada a Medida de Independência Funcional (MIF) neste momento. 
Seguimento foi realizado no hospital (quando paciente ainda se encontrava internado) ou na casa do participante pelos entrevistadores. Devido a casos de pessoas encaminhadas para tratamento em Manaus, foi realizada busca ativa destes casos no principal hospital de referência da capital, com finalidade de seguimento do desfecho.

A definição de caso foi a empregada pela Organização Mundial da Saúde: "rebaixamento neurológico focal (ou global) de início súbito que persiste por mais de 24 horas (ou conduz a morte antes desse tempo) de provável origem vascular". ${ }^{4}$ Os episódios isquêmicos transitórios não foram considerados no estudo. Não foi realizada definição quanto ao subtipo do AVC por causa da inexistência de exames complementares no município, como a tomografia computadorizada, sendo o diagnóstico estabelecido apenas pelo exame clínico.

O EMMA-Coari foi aprovado pelo Comitê de Ética em Pesquisa da Universidade Federal do Amazonas (UFAM) com CAAE no. 0111.0.115.000-10. Todos os participantes da pesquisa forneceram o termo de consentimento livre $e$ esclarecido, em duas vias, sendo respeitadas as normas de pesquisa envolvendo seres humanos com base a partir da Resolução $n^{\circ}$ 466/12 do Conselho Nacional de Saúde.

Os dados foram apresentados como estatística descritiva. As variáveis idade, tempo de internação e MIF foram apresentadas em média e mediana. Dados sobre sexo, raça, escolarização, renda mensal, condições pré-clínicas e status vital foram apresentados como valor absoluto e porcentagem. Nas evoluções clínicas, o NIHSS foi apresentado como média e mediana de todos os participantes, além do número absoluto e porcentagem, conforme as categorias da escala: $0-5$, sem comprometimento; 6-15, comprometimento leve, e 16-42, comprometimento grave. A mRS está apresentada em valores absolutos e porcentagem, conforme as categorias: sem dependência (0-1), dependência moderada (2-3), dependência grave (4-5) e morte (6). Foram calculadas também a incidência hospitalar (casos hospitalares / população total no período* 100.000) e as taxas de letalidade (número de óbitos pela doença / total de casos da doença $* 100$ ), e seus respectivos intervalos de $95 \%$ de confiança (IC $95 \%$,), no 10 e 28 dias pósAVC.

\section{RESULTADOS}

Em um ano de observação, a amostra hospitalar foi composta por 23 pacientes com diagnóstico clínico de AVC, com uma incidência hospitalar de 30,0 casos por 100.000 habitantes (IC $95 \%, 20,0-45,0)$. Todos os participantes foram seguidos em relação ao seu status vital, no entanto não foi possível realizar avaliação funcional em 2 pessoas transferidas para o município de Manaus-AM.

Os indivíduos da amostra tiveram idade média de 72,8 (DP= $12,4)$ anos, sendo em sua maioria homens $(65,2 \%)$, pardos $(65,2 \%)$, sem estudo formal $(60,9 \%)$ e com renda mensal média entre 1 e 2 salários-mínimos (56,6\%). Quanto às condições préclínicas relatadas pelos participantes, destaca-se a alta prevalência de hipertensão $(95,7 \%)$ e diabetes $(47,8 \%)$ entre os casos (Tabela 1). O tempo entre o evento e a admissão no HRC foi de até 24 horas em $82,6 \%$ dos casos. A amostra teve mediana de 28 pontos (intervalo interquartílico, $I \mathrm{QQ}=15-38$ ) na escala NIHSS, sendo a maioria com grave comprometimento neurológico (73,9\%) na admissão, e a mediana do tempo de internação hospitalar foi de 5 dias $(I I Q=3-9)$. As taxas de letalidades no 10 e e 28 ㅇa dias após o AVC foram de $30,4 \%$ (IC $95 \%$, $11,6-49,2)$ e $34,8 \%$ (IC95\%, 15,3-54,3), respectivamente.

Tabela 1. Perfil dos casos de AVC coletados no Step 1 na cidade de Coari, Amazonas, quanto a aspectos sociodemográficos e pré-clínicos

\begin{tabular}{|c|c|c|}
\hline CARACTERÍSTICAS & $\mathbf{N}$ & $\%$ \\
\hline \multicolumn{3}{|l|}{ Sociodemográficas } \\
\hline Idade ${ }^{a}$ & $N=23$ & 100 \\
\hline Média (DP) & \multicolumn{2}{|c|}{$72,8(12,4)$} \\
\hline Mediana (IIQ) (min-máx.) & \multicolumn{2}{|c|}{$73(60-86)(50-88$} \\
\hline \multicolumn{3}{|l|}{ Sexo } \\
\hline Masculino & 15 & 65,2 \\
\hline Feminino & 8 & 34,8 \\
\hline \multicolumn{3}{|l|}{ Raça } \\
\hline Branco & 4 & 17,4 \\
\hline Pardo & 15 & 65,2 \\
\hline Negro & 4 & 17,4 \\
\hline \multicolumn{3}{|l|}{ Escolaridade } \\
\hline Sem estudo formal & 14 & 60,9 \\
\hline Fundamental incompleto (1-7 anos) & 6 & 26,1 \\
\hline Fundamental completo ( 8 anos) & 3 & 13 \\
\hline \multicolumn{3}{|l|}{ Renda mensal } \\
\hline Até 1 SM & 7 & 30,4 \\
\hline $1-2 \mathrm{SM}$ & 13 & 56,6 \\
\hline 2 ou mais SM & 3 & 13 \\
\hline \multicolumn{3}{|l|}{ Condições pré-clínicas* } \\
\hline Hipertensão & 22 & 95,7 \\
\hline Diabetes & 11 & 47,8 \\
\hline Cardiopatia & 3 & 13 \\
\hline Tabagista atual & 5 & 21,7 \\
\hline Consumo de álcool de risco ${ }^{+\dagger}$ & 4 & 17,4 \\
\hline
\end{tabular}

${ }^{a}$ Distribuição de idade aderente à Normal (teste de Kolmogorov-Smirnov); ${ }^{*}$ História clínica de acordo com os relatos dos participantes; ${ }^{++}$Consumo de risco; DP: desvio padrão; IIQ: intervalo inter-quartílico

Pela mRS observa-se elevada proporção de casos com dependência grave (42,9\%), em comparação àqueles sem dependência ou com dependência moderada (14,3\% e 9,5\%, respectivamente), no 10 dia após o AVC. Já em 28 dias após o AVC verifica-se uma diminuição dos casos com dependência grave $(23,8 \%)$ e mais casos sem dependência ou com dependência moderada ( $23,8 \%$ e $14,3 \%$, respectivamente), o que sugere certa evolução funcional. Em 28 dias após o evento, os sobreviventes obtiveram pontuação mediana de 49 (IIQ=1891) pela MIF (Tabela 2).

\section{DISCUSSÃO}

O Step 1 (fase hospitalar) do EMMA-Coari permitiu alguns conhecimentos sobre características de admissões, gravidade da doença e aspectos funcionais no período estudado para o município. Em geral, os pacientes internados tiveram grave comprometimento neurológico no período de admissão (acima de $70 \%$ da amostra). Estudos brasileiros que utilizam o NIHSS apresentaram grande variabilidade dos achados. Estudos realizados na região nordeste do Brasil tiveram média de 13 pontos na NIHSS em até 90 dias após o evento. ${ }^{15,16}$ Enquanto estudos na região sul do Brasil demonstram pontuação na internação hospitalar de 5 a 8 pontos, e na alta hospitalar (cerca de 12 dias após internação) fica entre 2 e 6 pontos. ${ }^{17,18}$ 
Tabela 2. Perfil dos casos de AVC coletados no Step 1 na cidade de Coari, Amazonas, quanto a aspectos relativos à evolução clínico-funcional

\begin{tabular}{|c|c|c|}
\hline Evolução clínico-funcional & $\mathbf{N}$ & $\%$ \\
\hline \multicolumn{3}{|l|}{ NIHSS na admissão } \\
\hline Média (DP) & \multicolumn{2}{|c|}{$25,0(14,2)$} \\
\hline Mediana (IIQ) (min-máx.) & \multicolumn{2}{|c|}{$28(15-38)(0-41)$} \\
\hline $0-5$ & 4 & 17,4 \\
\hline $6-15$ & 2 & 8,7 \\
\hline $16-42$ & 17 & 73,9 \\
\hline \multicolumn{3}{|c|}{ Tempo entre o AVC e admissão no hospital } \\
\hline 0 dia & 19 & 82,6 \\
\hline 1 ou mais dias & 4 & 17,4 \\
\hline \multicolumn{3}{|l|}{ Tempo de internação no hospital } \\
\hline Média (DP) & \multicolumn{2}{|c|}{$6,6(5,1)$} \\
\hline Mediana (IIQ) (min-máx.) & \multicolumn{2}{|c|}{$5(3-9)(1-18)$} \\
\hline \multicolumn{3}{|l|}{ Status vital em 10 dias após AVC } \\
\hline Vivo & 16 & 69,6 \\
\hline Morto & 7 & 30,4 \\
\hline \multicolumn{3}{|l|}{ Rankin em 10 dias após AVC $(n=21)$} \\
\hline Sem dependência (0-1) & 3 & 14,3 \\
\hline Dependência moderada (2-3) & 2 & 9,5 \\
\hline Dependência grave (4-5) & 9 & 42,9 \\
\hline Morte (6) & 7 & 33,3 \\
\hline \multicolumn{3}{|l|}{ Status vital em 28 dias após AVC } \\
\hline Vivo & 15 & 65,2 \\
\hline Morto & 8 & 34,8 \\
\hline \multicolumn{3}{|c|}{ Rankin em 28 dias após AVC $(n=21)$} \\
\hline Sem dependência (0-1) & 5 & 23,8 \\
\hline Dependência moderada (2-3) & 3 & 14,3 \\
\hline Dependência grave (4-5) & 5 & 23,8 \\
\hline Morte (6) & 8 & 38,1 \\
\hline \multicolumn{3}{|l|}{ MIF em 28 dias $(n=13)$} \\
\hline Média (DP) & 57, & \\
\hline Mediana (IIQ) (min-máx.) & $49(18$ & \\
\hline
\end{tabular}

DP: desvio padrão; IIQ: intervalo inter-quartílico; NIHSS: National Institutes of Health Stroke Scale; MIF: Medida de Independencia Funcional

No entanto, no hospital de Coari foi encontrada elevada pontuação (mediana de 28 pontos). Uma possível explicação para essa disparidade entre as regiões pode ser devido a diferentes metodologias dos estudos. Tanto o presente estudo quanto aqueles da região nordeste não se limitaram a um subtipo de AVC em suas amostras, enquanto os estudos da região sul avaliaram somente pacientes com AVC isquêmico. Apesar de ser menos comum, o AVC hemorrágico costuma ser mais severo, ${ }^{1,19}$ e pode impactar em alguma medida em elevadas pontuações pelo NIHSS.

Outra possível explicação pode estar nas diferenças socioeconômicas e dos recursos estruturais hospitalares entre as cidades e regiões brasileiras. A tomografia computadorizada (TC) é uma ferramenta utilizada para melhor definição diagnóstica na doença cerebrovascular; entretanto não está disponível no Hospital Regional de Coari, enquanto fica disponível 24 horas por dia em todos hospitais regionais de Joinville/SC, por exemplo. ${ }^{20,21}$

Apesar da escala NIHSS não ser recomendada para pacientes crônicos, ${ }^{22}$ alguns estudos a utilizaram e revelaram um comportamento semelhante da pontuação entre regiões. Estudos em Natal/RN observaram uma média de 5 pontos, ${ }^{23,24}$ enquanto um estudo em Campinas/SP observou uma média de pontos de 1,61 em pacientes com AVC crônico. ${ }^{25} \mathrm{Em}$ outros países, temos: um estudo de base hospitalar realizado em
Taiwan que encontrou, em 360 indivíduos com primeiros episódios de AVC isquêmico (idade média de 64,9 anos $(\mathrm{DP}=12,5))$, uma proporção de $19,4 \%$ de pacientes graves nas primeiras 48 horas, $^{26}$ e proporção semelhante (20\%) encontrada por Dalal et al. ${ }^{27}$ entre 350 pacientes hospitalares com AVC primo em Mumbai, Índia (idade média de 66 anos $(D P=13,6)) .^{27}$

Um estudo multicêntrico europeu observou em uma amostra de 101 indivíduos a mediana de 11 pontos na escala NIHSS em pacientes admitidos pelo primeiro episódio de AVC isquêmico, ${ }^{28}$ enquanto um estudo nos Estados Unidos, com amostra de 317 indivíduos internados por AVC, observou uma pontuação média entre 7,17 a 9,33 na admissão dependendo da lateralidade do evento. ${ }^{29}$ Conforme esperado, os pacientes tiveram baixa média de MIF e alta letalidade em 28 dias após a doença, evidenciando estreita relação inversa entre comprometimento neurológico e prognóstico funcional. ${ }^{15} \mathrm{Um}$ estudo de Belo Horizonte/MG, com pacientes de AVC isquêmico recém admitidos em um hospital, observou média de $113,67(\mathrm{DP}=12,44)$ na $\mathrm{MIF}^{30}$

Uma MIF média de 58 (DP= 27) foi encontrada na admissão à unidade de reabilitação no hospital SARAH em Brasília/DF em 252 pacientes de AVC, com variação de 1 a 12 meses do evento. ${ }^{31} \mathrm{Em}$ um estudo realizado em Natal foi observado uma média de 54,6 (DP= 17,2) na MIF motora - versão menor da $\mathrm{MIF}$, excluindo o componente cognitivo. ${ }^{15} \mathrm{E}$ em São Carlos/SP a MIF Motora média foi de 56,83 ( $D P=24,99)$ em pacientes de AVC com, em média, 12 meses do evento. ${ }^{32}$ Em Santa Maria/RS, um estudo observou que $60 \%$ dos pacientes hospitalizados por AVC tinha um grave comprometimento funcional quando avaliados pela mRS. ${ }^{14}$

Há de se considerar que, a exemplo do que ocorreu com a evolução funcional na amostra medido pela mRS, o nível de independência funcional, ou outra medida de incapacidade física, tende a melhorar com o tempo após a lesão, tanto pela evolução clínica da doença quanto pelo viés de sobrevida (os mais graves morrem mais que os menos comprometidos), e que a reabilitação exerce forte impacto no aumento deste nível, como demonstrado em estudos anteriores .

Estudos brasileiros anteriores, com informações sobre letalidade por AVC, mostraram taxas variando de $10,4 \%$ a 20,9\% em 1 mês após o evento. ${ }^{33-36}$ Um outro estudo de 2012, realizado em alguns centros hospitalares no Brasil, demonstrou taxas de letalidade hospitalar por AVC, em 28 dias após o evento, de 8,4\% em Natal/RN, 24,3\% em João Pessoal/PB e de $13,5 \%$ em São Paulo/SP, ${ }^{37}$ todas ajustadas por idade; no entanto os autores não encontraram diferença estatisticamente significativa entre as localidades.

Estudos mais recentes revelaram em Joinville/SC uma letalidade entre $22,5 \%$ e $26,5 \%$ em pacientes com AVC isquêmico, ${ }^{17}$ e em São Paulo/SP uma proporção de 12,8\%, ambos em 30 dias de seguimento. ${ }^{19}$ Portanto, proporções acima de $30 \%$ de óbito em casos de AVC ainda no primeiro mês - Step 1 de Coari - confere letalidade acima daquelas notificadas em outras regiões e hospitais do Brasil. A amostra hospitalar apresentou elevada média de idade (72,8 anos e DP= 12,4), quando comparados aos estudos citados, os quais tiveram médias de idade variando de 58 a 68 anos. A idade está diretamente associada a taxas de incidência, ao comprometimento neurológico no AVC e, consequentemente, 
ao prejuízo funcional e letalidade. ${ }^{38,39}$

Desta forma, a idade avançada parece ser uma das possíveis explicativas para diferenças entre as populações analisadas; apesar deste fator por si não ser suficiente para explicar tamanha discrepância encontrada, sobretudo para valores do NIHSS em comparação a estudos em outros países. Outros fatores com possível associação aos desfechos são o baixo nível socioeconômico da amostra e altas prevalências de hipertensão e diabetes, podendo estas também ter estreita relação com a idade avançada.

Neste sentido, é provável que aspectos relativos à rotina do hospital estudado estejam influenciando os resultados. Tendo em vista o número restrito de leitos para um hospital de referência regional, pode-se esperar priorização de internação para os pacientes mais graves, os quais tendem a ser os mais velhos. A experiência na coleta do Step 1 já apontava para tal fato, que parece confirmado pelos resultados.

Um aspecto que deve ser considerado é a qualidade do cuidado ofertado. As diferenças socioeconômicas regionais evidenciam a disparidade entre os recursos de estruturas hospitalares; estas podem ser identificadas pela deficiência de ambientes adequados e disponibilidade de equipamentos, e ainda por inadequações de recursos humanos e a taxa de rotatividade de funcionários. ${ }^{20}$ Hospitais com maiores volumes de pacientes e localizados em regiões de maior acesso a tecnologias médicas tendem a prestar melhor assistência e ter taxas de letalidades por AVC menores, em comparação a hospitais de pequeno porte, como visto por Ogbu et al. ${ }^{40}$ na Holanda. Um marcador nesta relação de cuidado hospitalar é justamente o uso da TC, como já comentado anteriormente.

Rolim e Martins ${ }^{41}$ avaliaram a relação entre taxas de mortalidade hospitalar (indicador de cuidado) por AVC isquêmico e uso de tomografia computadorizada no Brasil, considerando o perfil de gravidade dos casos, a partir de dados colhidos do Sistema de Informações Hospitalares do SUS (SIHSUS) em 2006 e 2007. As autoras encontraram que as hospitalizações com uso de TC forneceram efeito protetor para a mortalidade hospitalar (até 7 dias de internação) em relação àquelas sem uso de TC, mesmo após ajustes para idade, sexo e comorbidades relacionadas. A taxa de mortalidade hospitalar bruta foi de $15,5 \%$ no grupo que realizou TC e $41,8 \%$ no grupo que não realizou o exame.

O mesmo estudo revelou, também, a disparidade regional de disponibilidade e uso do instrumento diagnóstico, com concentração de $91,1 \%$ dos exames feitos nas internações nas regiões sudeste e sul do país. Os hospitais com melhor qualidade de desempenho (tercis pela razão entre mortalidade observada e esperada) tiveram maior volume de pacientes e menores taxas de mortalidade bruta em comparação com aqueles de menores qualidade de desempenho.

Neste sentido, apesar do presente estudo não ter realizado avaliação direta da qualidade da assistência hospitalar, é possível que a qualidade da assistência hospitalar em Coari, quer por carência tecnológica-estrutural, quer por baixo nível de cuidado da equipe de saúde do hospital, esteja relacionada às altas taxas de letalidade por AVC encontradas, em comparação aos demais estudos brasileiros realizados em hospital - todos com maior volume de pacientes e com melhor acesso à tecnologia médica, como uso da TC. Estudos na Holanda ${ }^{40}$ e Canadá ${ }^{39}$ com grande contingente populacional e com médias de idade similares à encontrada na amostra hospitalar de Coari apresentaram menores letalidades em 30 dias da doença ( $26 \%$ e $12,6 \%$, respectivamente). Vale salientar que o estudo holandês abrangeu todos os hospitais do país, incluindo os de pequeno volume, similar ao estudo brasileiro de Rolim e Martins, ${ }^{41}$ mas ainda com taxas menores do que este.

Nestes países é possível encontrar, em grande escala, elevado nível de assistência ao paciente com AVC, como a incorporação de unidades de AVC (caráter multiprofissional e especializado para tratamento e reabilitação precoce da doença, sob critérios de excelência terapêutica), com forte influência na melhoria de desfechos funcionais e de mortalidade precoce; ${ }^{42,43}$ além de se tratar de populações com níveis socioeconômicos mais elevados que as amazônicas. Tal hipótese necessita de futuras investigações para sua validação.

Como o presente estudo teve como foco a vigilância epidemiológica do AVC, através de metodologia com base na versão simplificada da WHO STEPS Stroke (Step 1), informações como subtipo da doença não foram abordados, mesmo para os eventos hospitalizados, devido à prática diagnóstica no Hospital Regional de Coari ser centrada apenas na clínica médica.

Ainda assim o número de 23 casos em um ano de observação nos revela uma incidência hospitalar de $30 \mathrm{em}$ 100.000 habitantes; contudo, dados do DATASUS apontam para 18 casos de AVC para o mesmo período. Esta diferença entre os dados oficiais e o que foi coletado indica baixa qualidade de registros hospitalares na época no município.

A taxa encontrada parece alta em relação à média de hospitalizações por AVC na região Norte e no Brasil (13,0 e 8,8 por 100.000 habitantes, respectivamente).${ }^{44}$ No entanto, devese lembrar que limitações como o baixo número de admissão e o viés de aferição podem estar presentes neste estudo, e tais limitações reduzem o poder de comparabilidade destes dados com os de outros estudos, mesmo para aqueles com metodologias similares.

O Manual do STEPS Stroke recomenda como ideal, para se alcançar estimativas confiáveis do impacto da doença com o uso deste método, populações de 250.000 até 1.000 .000 de pessoas, com vistas a minimizar os vieses de excessivo ou baixo contingente populacional e de casos. ${ }^{4}$ A despeito disto, os dados hospitalares coletados servem como parâmetro de validade interna para o município em análise e nos fornece informações relevantes a respeito do cuidado a estes pacientes.

\section{CONCLUSÃO}

O Step 1 do Estudo EMMA-Coari revelou uma predominância de homens, com idade elevada, pardos, sem estudo formal, baixa renda e com histórico de hipertensão arterial e diabetes, como perfil de internação hospitalar por doenças cerebrovasculares no município. A incidência de casos hospitalares de AVC foi em 30 (por 100.000 habitantes) em Coari-AM, com graves comprometimentos neurológicos na admissão, e altas taxas de letalidade e prejuízo funcional após 28 dias do evento, quando comparadas a outras localidades no Brasil e no mundo. É possível que investimento na melhoria da qualidade do cuidado, envolvendo acurácia diagnóstica, 
tecnologia e capacitação da assistência, reabilitação precoce e multiprofissional tenha papel importante para melhoria das condições de saúde de pessoas com AVC na região.

\section{AGRADECIMENTOS}

Os pesquisadores gostariam de agradecer ao CNPq pelo suporte financeiro e à equipe de coleta de dados envolvida no estudo. Este estudo foi financiado pelo Edital MCT/CNPq N $~ o$ 14/2009 - Universal, processo no 475439/2009-0 do Conselho Nacional de Desenvolvimento Científico e Tecnológico (CNPq).

\section{REFERÊNCIAS}

1. Katan M, Luft A. Global Burden of Stroke. Semin Neurol. 2018;38(2):208-211. Doi: https://doi.org/10.1055/s-0038 1649503

2. Bensenor IM, Goulart AC, Szwarcwald CL, Vieira ML, Malta DC, Lotufo PA. Prevalence of stroke and associated disability in Brazil: National Health Survey--2013. Arq Neuropsiquiatr. 2015;73(9):746-50. Doi: https://doi.org/10.1590/0004-282X20150115

3. WHO. Disability: Report by the secretariat [text on the Internet]. Geneva: WHO; c2013 [cited 2020 Aug 12]. Available

from: https://apps.who.int/gb/ebwha/pdf files/EB132/B132 1 0-en.pdf

4. World Health Organization. The WHO STEPwise approach to stroke surveillance. Geneva: WHO; 2005.

5. Truelsen T, Bonita R, Jamrozik K. Surveillance of stroke: a global perspective. Int J Epidemiol. 2001;30 Suppl 1:S116. Doi: https://doi.org/10.1093/ije/30.suppl 1.s11

6. IBGE. Censo demográgico 2010 [texto na Internet]. Rio de Janeiro: IBGE; c2010 [citado 2020 Ago 12]. Disponível em: https://cidades.ibge.gov.br/brasil/am/coari/panorama

7. Fernandes TG, Benseñor IM, Goulart AC, Tavares BM, Alencar AP, Santos IS, et al. Stroke in the rain forest: prevalence in a ribeirinha community and an urban population in the Brazilian Amazon. Neuroepidemiology. 2014;42(4):235-42.

Doi: https://doi.org/10.1159/000362210

8. Branco JP, Oliveira S, Sargento-Freitas J, Laíns J, Pinheiro J. Assessing functional recovery in the first six months after acute ischemic stroke: a prospective, observational study. Eur J Phys Rehabil Med. 2019;55(1):1-7. Doi: https://doi.org/10.23736/S1973-9087.18.05161-4

9. Musa KI, Keegan TJ. The change of Barthel Index scores from the time of discharge until 3-month post-discharge among acute stroke patients in Malaysia: A random intercept model. PLoS One. 2018;13(12):e0208594. Doi: https://doi.org/10.1371/journal.pone.0208594

10. Kelly-Hayes $M$, Beiser A, Kase CS, Scaramucci A, D'Agostino RB, Wolf PA. The influence of gender and age on disability following ischemic stroke: the Framingham study. J Stroke Cerebrovasc Dis. 2003;12(3):119-26. Doi: https://doi.org/10.1016/S1052-3057(03)00042-9

11. Longley V, Peters S, Swarbrick C, Rhodes S, Bowen A. Does pre-existing cognitive impairment impact on amount of stroke rehabilitation received? An observational cohort study. Clin Rehabil. 2019;33(9):1492-1502. Doi: https://doi.org/10.1177/0269215519843984
12. Meyer MJ, Pereira S, McClure A, Teasell R, Thind A, Koval $\mathrm{J}$, et al. A systematic review of studies reporting multivariable models to predict functional outcomes after post-stroke inpatient rehabilitation. Disabil Rehabil. 2015;37(15):1316-23.

Doi: https://doi.org/10.3109/09638288.2014.963706

13. Goulart AC, Bustos IR, Abe IM, Pereira AC, Fedeli LM, Benseñor IM, et al. A stepwise approach to stroke surveillance in Brazil: the EMMA (Estudo de Mortalidade e Morbidade do Acidente Vascular Cerebral) study. Int J Stroke. 2010;5(4):284-9. Doi: https://doi.org/10.1111/i.1747-4949.2010.00441.x

14. Trevisan CM, Marcon CLV, Cavalheiro BR, Melo LP, Campos TF. Aspectos clínicos relevantes de pacientes com acidente vascular cerebral na emergência hospitalar: implicações para o serviço público de saúde. Rev Ciênc Méd Biol. 2015;14(2):171-6. Doi: http://dx.doi.org/10.9771/cmbio.v14i2.13607

15. Costa FA, Silva DLA, Rocha VM. Severidade clínica e funcionalidade de pacientes hemiplégicos pós-AVC agudo atendidos nos serviços públicos de fisioterapia de Natal (RN). Cien Saude Colet. 2011;16 (S1):1341-8. Doi: https://doi.org/10.1590/S1413-81232011000700068

16. Costa FA, Silva DLA, Rocha VM. Estado neurológico e cognição de pacientes pós-acidente vascular cerebral. Rev Esc Enferm USP. 2011 Rev Esc Enferm USP. 2011;45(5):1083-8. Doi: https://doi.org/10.1590/S0080$\underline{62342011000500008}$

17. Amaral CHD, Amaral AR, Nagel V, Venancio V, Garcia AC, Magalhaes PS, et al. Incidence and functional outcome of atrial fibrillation and non-atrial fibrillation- related cardioembolic stroke in Joinville, Brazil: a populationbased study. Arq Neuropsiquiatr. 2017;75(5):288-94. Doi: https://doi.org/10.1590/0004-282X20170039

18. Hanauer L, Schmidt D, Miranda RE, Borges MK. Comparação da severidade do déficit neurológico de pacientes com acidente vascular cerebral isquêmico agudo submetidos ou não à terapia trombolítica. Fisioter Pesq. 2018;25(2):217-23. Doi: https://doi.org/10.1590/1809-2950/18020425022018

19. Goulart AC, Bensenor IM, Fernandes TG, Alencar AP, Fedeli LM, Lotufo PA. Early and one-year stroke case fatality in Sao Paulo, Brazil: applying the World Health Organization's stroke STEPS. J Stroke Cerebrovasc Dis. 2012;21(8):832-8.

20. Machado JP, Martins ACM, Martins MS. Avaliação da qualidade do cuidado hospitalar no Brasil: uma revisão sistemática. Cad Saude Publica. 2013;29(6):1063-82. Doi: https://doi.org/10.1590/s0102-311x2013000600004

21. Lange $\mathrm{MC}$, Cabral NL, Moro $\mathrm{CH}$, Longo $\mathrm{AL}$, Gonçalves $\mathrm{AR}$, Zétola VF, Rundek T. Incidence and mortality of ischemic stroke subtypes in Joinville, Brazil: a population-based study. Arq Neuropsiquiatr. 2015;73(8):648-54. Doi: https://doi.org/10.1590/0004-282X20150081

22. StrokEDGE II Task Force. Final EDGE recommendations [text on the Internet]. Minneapolis: Academy of Neurologic Physical Therapy; c2018 [cited 2020 Aug 19]. Available from: https://www.neuropt.org/practiceresources/neurology-section-outcome-measuresrecommendations/stroke 
23. Campos TF, Dantas AATSG, Protásio de Melo L, Carvalho de Oliveira D. Grau neurológico e funcionalidade de pacientes crônicos com acidente vascular cerebral: Implicações para a prática clínica. Arq Ciênc Saúde. 2014;21(1):28-33.

24. Dantas AATSG, Torres SVS, Farias IMA, Sant'Ana SBCL, Campos TF. Rastreio cognitivo em pacientes com acidente vascular cerebral: um estudo transversal. J Bras Psiquiatr. 2014;63(2):98-103. Doi: https://doi.org/10.1590/0047$\underline{2085000000012}$

25. Yoshida HM, Lima FO, Fernandes PT. Habilidade motora e hemisfério cerebral em pacientes pós-AVC: existe relação? Rev Bras Neurol Psiquiatr. 2019;23(3):196-205.

26. Tseng MC, Chang KC. Stroke severity and early recovery after first-ever ischemic stroke: results of a hospital-based study in Taiwan. Health Policy. 2006;79(1):73-8. Doi: https://doi.org/10.1016/j.healthpol.2005.12.003

27. Dalal PM, Malik S, Bhattacharjee M, Trivedi ND, Vairale J, Bhat $\mathrm{P}$, et al. Population-based stroke survey in Mumbai, India: incidence and 28-day case fatality. Neuroepidemiology. 2008;31(4):254-61. Doi: https://doi.org/10.1159/000165364

28. Cheng B, Forkert ND, Zavaglia M, Hilgetag CC, Golsari A, Siemonsen $S$, et al. Influence of stroke infarct location on functional outcome measured by the modified rankin scale. Stroke. 2014;45(6):1695-702. Doi: https://doi.org/10.1161/STROKEAHA.114.005152

29. Hedna VS, Bodhit AN, Ansari S, Falchook AD, Stead L, Heilman KM, et al. Hemispheric differences in ischemic stroke: is left-hemisphere stroke more common? J Clin Neurol. 2013;9(2):97-102. Doi: https://doi.org/10.3988/jcn.2013.9.2.97

30. Pedroso VSP, Brunoni AR, Vieira ÉLM, Jorge RE, Lauterbach EC, Teixeira AL. Early psychiatric morbidity in a Brazilian sample of acute ischemic stroke patients. Clinics (Sao Paulo). 2018;73:e55. Doi: https://doi.org/10.6061/clinics/2018/e055

31. Carod-Artal FJ, Medeiros MS, Horan TA, Braga LW. Predictive factors of functional gain in long-term stroke survivors admitted to a rehabilitation programme. Brain Inj. 2005;19(9):667-73. Doi: https://doi.org/10.1080/02699050400013626

32. Caro CC, Cruz DMC. Correlação entre independência funcional e cognição em homens com AVC. Rev Ter Ocup Univ São Paulo. 2017;28(2):173. Doi: https://doi.org/10.11606/issn.2238-6149.v28i2p173-180

33. Minelli C, Fen LF, Minelli DPC. Stroke incidence, prognosis, 30-day, and 1-year case fatality rates in Matão, Brazil: A population-based prospective study. Stroke. 2007;38(11):2906-11.

Doi:
34. Cabral NL, Gonçalves AR, Longo AL, Moro CH, Costa G, Amaral $\mathrm{CH}$, et al. Incidence of stroke subtypes, prognosis and prevalence of risk factors in Joinville, Brazil: a 2 year community based study. J Neurol Neurosurg Psychiatry. 2009;80(7):755-61.

https://doi.org/10.1136/innp.2009.172098

35. Martins Jr AN, Figueiredo MM, Rocha OD, Fernandes MA, Jeronimo SM, Dourado Jr ME. Frequency of stroke types at an emergency hospital in Natal, Brazil. Arq Neuropsiquiatr. 2007;65(4B):1139-43. Doi: https://doi.org/10.1590/s0004-282×2007000700009

36. Carvalho JJ, Alves MB, Viana GÁ, Machado CB, Santos BF, Kanamura $A H$, et al. Stroke epidemiology, patterns of management, and outcomes in Fortaleza, Brazil: a hospital-based multicenter prospective study. Stroke. 2011;42(12):3341-6.

Doi: https://doi.org/10.1161/STROKEAHA.111.626523

37. Fernandes TG, Goulart AC, Campos TF, Lucena NM, Freitas $\mathrm{KL}$, Trevisan $\mathrm{CM}$, et al. Early stroke case-fatality rates in three hospital registries in the Northeast and Southeast of Brazil. Arq Neuropsiquiatr. 2012;70(11):869-73. Doi: https://doi.org/10.1590/s0004-282x2012001100009

38. Macciocchi SN, Diamond PT, Alves WM, Mertz T. Ischemic stroke: relation of age, lesion location, and initial neurologic deficit to functional outcome. Arch Phys Med Rehabil. 1998;79(10):1255-7. Doi: https://doi.org/10.1016/s00039993(98)90271-4

39. Saposnik G, Hill MD, O'Donnell M, Fang J, Hachinski V, Kapral MK. Variables associated with 7-day, 30-day, and 1-year fatality after ischemic stroke. Stroke. 2008;39(8):2318-24. Doi: https://doi.org/10.1161/STROKEAHA.107.510362

40. Ogbu UC, Slobbe LC, Arah OA, Bruin A, Stronks K, Westert GP. Hospital stroke volume and case-fatality revisited. Med Care. 2010;48(2):149-56. Doi: https://doi.org/10.1097/MLR.0b013e3181bd4df1

41. Rolim CLRC, Martins M. Qualidade do cuidado ao acidente vascular cerebral isquêmico no SUS. Cad Saude Publica. 2011;27(11):2106-2116. Doi: https://doi.org/10.1590/s0102-311×2011001100004

42. Seenan $P$, Long $M$, Langhorne $P$. Stroke units in their natural habitat: systematic review of observational studies. Stroke. 2007;38(6):1886-92.

https://doi.org/10.1161/STROKEAHA.106.480871

Doi:

43. Meretoja A, Roine RO, Kaste $M$, Linna $M$, Roine $S$, Juntunen $M$, et al. Effectiveness of primary and comprehensive stroke centers: PERFECT stroke: a nationwide observational study from Finland. Stroke. 2010;41(6):1102-7. Doi: https://doi.org/10.1161/STROKEAHA.109.577718

44. Kurtz P, Bastos LS, Aguilar S, Hamacher S, Bozza FA. Effect of seasonal and temperature variation on hospitalizations for stroke over a 10-year period in Brazil. Int J Stroke. 2020:1747493020947333.

Doi: 